\title{
ANÁLISIS REFLEXIVO DEL CUIDADO EN REFLEXOLOGÍA Y MASOTERAPIA CENTRADO EN LA PERSONA, POR PARTE DEL PROFESIONAL DE ENFERMERÍA
}

\author{
REFLEXIVE ANALYSIS IN REFLEXOLOGY AND MASSOTHERAPY \\ CLIENT-CENTRED CARE, BY THE NURSING PROFESSION
}

\author{
BÁltica Cabieses V. \\ Sarah Mary Miner ** \\ Natalia Villegas R. ${ }^{* * *}$
}

\begin{abstract}
RESUMEN
Las terapias alternativas y complementarias (TAC) constituyen parte importante de las intervenciones desarrolladas en salud y solicitadas por quienes buscan resolver algún problema, mantener o mejorar su situación de salud y bienestar. El presente estado del arte tiene como propósito dar a conocer el análisis reflexivo del cuidado centrado en la persona e integral que surge de la realización de reflexología y masoterapia por parte del profesional de Enfermería. Primero, se desarrolla una descripción de diversas definiciones existentes en torno a TAC, luego se describe la conexión existente entre el cuidado alternativo y complementario y la ciencia de Enfermería, a continuación se desarrolla el proceso de cuidado en masoterapia y reflexología, posteriormente se exponen a través de una discusión los elementos centrales del cuidado centrado en la persona, pilar esencial de las TAC, y finalmente se establecen las conclusiones e implicancias para la práctica de Enfermería de estas terapias.
\end{abstract}

Palabras clave: Enfermería, cuidado centrado en la persona, terapias alternativas, reflexología, masoterapia.

\begin{abstract}
Complementary and alternative therapies (CAT) represent a relevant dimension of care in the Nursing practice. They are requested by people who seek to resolve a particular health problem or condition, and to improve their wellness and health perception. This article is a state of the art and has the purpose of sharing the reflexive analysis of alternative and complementary person-centered care, which emerges from the practice of massage and reflexology for several years by nurse professionals. Primarily, it describes the various definitions existing for complementary and alternative therapies, and then describes the connection among alternative-complementary care and the Nursing science. Afterwards, it develops the nursing process for massage and reflexology, then discusses the main aspects of person-centered care, most important factor in CAT, and finally establishes conclusions and suggestions for the Nursing practice towards these therapies.
\end{abstract}

Key words: Nursing, client-centred care, alternative therapies, reflexology, massotherapy.

Fecha recepción: 04/01/08 Fecha aceptación: 25/01/10

\footnotetext{
* Enfermera-matrona, Magíster en Epidemiología, docente investigador Universidad del Desarrollo-Clínica Alemana. Email:bcabieses@udd.cl

${ }^{* *}$ Enfermera, Magíster en Enfermería. Pontificia Universidad Católica de Chile. Email:smminer@uc.cl

${ }^{* * *}$ Enfermera-matrona, Magíster en Enfermería. Pontificia Universidad Católica de Chile. Email:navilleg@uc.cl
} 


\section{INTRODUCCIÓN: \\ HACIA UNA DEFINICIÓN DE TERAPIAS ALTERNATIVAS Y COMPLEMENTARIAS (TAC)}

Las terapias alternativas y complementarias (del inglés complementary and alternative therapies/medicine, CAT o CAM) constituyen actualmente parte importante de las intervenciones desarrolladas en salud y solicitadas por quienes buscan resolver algún problema, mantener o mejorar su situación de salud y bienestar. A nivel mundial, ha surgido un interés progresivo por este tipo de terapias durante las últimas décadas. En los Estados Unidos por ejemplo, de acuerdo a un reciente catastro nacional, el 36\% refiere haber utilizado algún tipo de terapia en los pasados 12 meses (1). Más aún, de acuerdo a lo descrito por Robb (2), estudios estiman que más del $50 \%$ de los norteamericanos participan en algún tipo de TAC, asumiendo gastos incluso millonarios por no estar contemplados en los seguros de salud. En Chile se desconoce la real magnitud de su uso, pero existe la percepción de un avance acelerado en los últimos años y que explicaría el creciente interés por la formación en torno a este tema.

Existen diversas definiciones para este concepto. En general, el término medicina alternativa designa de forma amplia los métodos y prácticas usados en lugar, o como complemento, de los tratamientos médicos referidos como convencionales para curar o amainar enfermedades. El alcance preciso de la medicina alternativa es objeto de cierto debate y depende en buena medida de lo que se entienda por medicina convencional. Sin embargo, éstas no sólo se utilizan en procesos de desviación de la salud, sino que también contribuyen al bienestar y la promoción de la salud. Así lo plantean Kim, Erlen, Kim y Sok (3) señalando que son "tanto tratamientos como estrategias de promoción de la salud diferentes a las propuestas por la medicina alopática y pueden contribuir a la satisfac- ción de las personas, a su bienestar y calidad de vida mediante la diversificación del concepto tradicional de prácticas de salud".

La Organización Mundial de la Salud (4, 5) por su parte, de acuerdo a su "Estrategia de la OMS sobre medicina tradicional 20022005", define las TAC como un término amplio utilizado para referirse tanto a los sistemas de Medicina Tradicional (MT), como por ejemplo la medicina tradicional china, el ayurveda hindú y la medicina unani árabe y las diversas formas de medicina indígena. Esta definición subraya la relevancia del concepto no alopático de las intervenciones, especialmente indígenas, pero no incorpora todas las alternativas existentes, cada día más numerosas y diversas.

La Asociación Española de Médicos Naturistas acuña las TAC bajo el concepto de Medicina Naturista y la define como un método terapéutico que tiene por base dos principios: el fisioterapéutico y el metodológico. En el primero se valora que la salud la mantiene el propio cuerpo, con sus propios sistemas y que en la enfermedad, son estos sistemas los que se ponen en marcha para la curación y reequilibrio; y en el segundo se consideran aquellas terapias basadas en remedios naturales, cuya aplicación ha dado lugar a técnicas reconocidas y probadas por su eficacia terapéutica, donde destaca el masaje terapéutico o masoterapia y la reflexología, entre otros (6).

La Oficina de Medicina Alternativa de los EE.UU. define las TAC como aquellos tratamientos y prácticas asistenciales que no se enseñan en las facultades de medicina, no se usan generalmente en los hospitales y no se reembolsan habitualmente por las compañías de seguros (7). No obstante, esta perspectiva se ha ido modificando, pues cada día más facultades y escuelas de educación superior incorporan esta línea de intervención sanitaria como herramienta útil y necesaria para el cuidado en la formación profesional de salud.

Los Institutos Nacionales de Salud de los 
EE.UU. (NIH) en un panel de definición y descripción del año 1997, señalan que las TAC son un dominio amplio de fuente de curación que integran todos los sistemas de salud con modalidades específicas de prácticas y un cuerpo propio de teorías y creencias, que son diferentes de aquellas que constituyen el sistema de salud de una sociedad en particular o cultura en un periodo de tiempo determinado. Esta definición considera tratamientos basados en la biología (hierbas, dietas especiales y vitaminas), tratamientos basados en la manipulación del cuerpo (masaje, quiropráctica, reflexología y osteopatía), terapias energéticas (reiki, terapia magnética, qi gong), tratamientos mente/cuerpo (yoga, espiritualidad, relajación y meditación) y completos sistemas médicos alternativos (medicina tradicional china, naturopatía, ayurveda) (8).

Otra interesante clasificación de las TAC corresponde a la de la British Medical Association (BMA), que las divide en cuatro: sistemas completos, métodos diagnósticos, métodos terapéuticos y de autocuidado. Los sistemas completos dicen relación con Homeopatía, Osteopatía, Quiropráctica, Medicina Herbal, Acupuntura y Naturopatía. Los métodos diagnósticos son Iridología, Kinesiología y Análisis capilar; los métodos terapéuticos corresponden a Masaje/terapias de contacto, Shiatsu, Reflexología, Aromaterapia, Medicina Nutricional e hipnosis; y los métodos de autocuidado incluyen Meditación, Yoga, Relajación y Tai-chi $(9,10)$.

El presente estado del arte surge de la inquietud de dar a conocer el análisis reflexivo del cuidado centrado en la persona e integral que ofrece la realización durante varios años de las terapias alternativas y complementarias. Cada día más, las enfermeras y los profesionales de la salud dedicados al cuidado de la persona se ven exigidos por la necesidad de perfeccionar y ampliar sus herramientas terapéuticas en pro de la recuperación del usuario, que supera el padecer puramente corporal e involucra al individuo de mane- ra completa y total. Las TAC en este sentido, estarían brindando perspectivas del cuidado y del proceso de curación interesantes e innovadoras que los mismos clientes tienden a solicitar con mayor frecuencia, como complemento a su terapia tradicional o alopática. El propósito del artículo es por lo tanto, desarrollar un análisis reflexivo del cuidado otorgado por el profesional de Enfermería en Reflexología y Masoterapia. En este sentido, pretende proporcionar elementos relevantes para situar Enfermería como apoyo en la práctica de algunas TAC, en particular al discutir el énfasis común de ambas prácticas, Enfermería y terapias alternativas, de estar centradas en la persona.

\section{USO DEL CUIDADO ALTERNATIVO Y COMPLEMENTARIO EN ENFERMERÍA}

El rol de Enfermería en las TAC no ha sido totalmente definido. Sin embargo, ya existen Escuelas de Enfermería que han tomado su posición al señalar que este tipo de intervenciones son consistentes con la práctica de Enfermería (3). De hecho, si se asume el desafío de buscar a través de Internet Escuelas de Enfermería que ofrezcan cursos de pre y postgrado en torno a TAC, el resultado es más exitoso y fecundo de lo esperado. La mayoría de los cursos, sin embargo, son de carácter optativo y un barniz introductorio a las diversas técnicas existentes, sin entregar herramientas sólidas para la práctica clínica de alguna de ellas en particular. Opinión similar tienen (11) quienes postulan que las enfermeras valoran las TAC, pero desconocen cómo aplicarlas. De esta manera, la educación pertinente en torno a estas podría incidir en el conocimiento y aplicación por parte de profesionales de Enfermería y otras ciencias de la salud. Los mismos autores consideran que la inclusión de la temática de TAC en el currículo de Enfermería es esporádica y que las instituciones de educación su- 
perior necesitan incorporarlos prontamente en la formación profesional en salud.

$\mathrm{Al}$ mismo tiempo, de acuerdo a un estudio del año 2006 (12), existe considerable interés por parte de los enfermeros por aprender con mayor profundidad sobre las TAC y han canalizado esta motivación encontrando formas diversas, informales y extrauniversitarias para hacerlo. Kim, Erlen, Kim y Sok (3) realizaron un estudio descriptivo en torno a conocimientos, experiencias y actitudes de estudiantes, académicos y profesionales de Enfermería sobre TAC, reportando que más del $70 \%$ de la muestra estaba de acuerdo con la integración de las TAC en la practica clínica y más del $85 \%$ deseaba mayor formación en este tema. Todos los grupos en estudio expresaron una actitud positiva hacia la educación profesional sobre estas terapias, aun cuando solo cerca del 30\% reportaron tener alguna experiencia realizándolas o recibiéndolas. El interés por este tipo de intervenciones se relacionaría con su carácter menos invasivo, no narcótico y de fácil acceso en comparación a otros tipos de terapias convencionales (2).

Visto de manera global, se podría decir que en muchos lugares las TAC siguen siendo una disciplina que se transmite por tradición oral, de expertos a discípulos que toman el riesgo de conocer filosofías y paradigmas del proceso de salud y enfermedad diferentes, complementarios y en algunas ocasiones fuertemente criticados por la débil evidencia científica disponible respecto de su eficacia. En este sentido, el año 2000 el Consejo de Enfermería y Matronería (NMC) advirtió que aquellos profesionales que practican alguna TAC debe asegurar y garantizar que son competentes para su realización. Este consejo agregó que estas terapias requerían ser seguras y utilizadas como parte de un proceso de cuidado, que considerara el consentimiento del usuario para su ejecución (13). Sin lugar a dudas las TAC tienen un largo camino por recorrer para ser aceptadas y especialmente validadas en el escenario de investigación, pero ya han comenzado un avance acelerado en el escenario clínico individual.

\section{EL CUIDADO EN REFLEXOLOGÍA Y MASOTERAPIA}

La masoterapia y reflexología son técnicas específicas en donde se trabajan partes blandas del organismo, en especial tejido muscular. La masoterapia se puede realizar en todo el cuerpo y la reflexología tiene la particularidad de proyectar en sitios específicos, como por ejemplo la planta de los pies, los órganos de todo el cuerpo. La reflexología se puede realizar también en manos y cuero cabelludo. Tanto la masoterapia como la reflexología tienen dos enfoques, uno de promoción de la salud -a favor del bienestar- y otro terapéutico -cuyo objetivo es sanar procesos de desviación de la salud-. A continuación se realizara una descripción del cuidado en reflexología y masoterapia, conforme a los lineamientos del proceso de atención de Enfermería $(14,15)$.

Respecto de la valoración de Enfermería en reflexología y masoterapia, destaca la motivación por la cual una persona solicita este tipo de intervenciones. De acuerdo a la experiencia recopilada durante 6 años por C. P., enfermera-matrona, reflexóloga y masoterapeuta entrevistada por las autoras de este artículo durante el año 2007en la zona sur de Chile, los principales motivos de consulta son de dos tipos, los de dolor corporal específico -que llega incluso a la indicación médica de "masaje terapéutico" - y los de búsqueda del bienestar. El dolor corporal dice relación con contracturas musculares y procesos traumáticos secundarios a sobreesfuerzo físico, característico de deportistas, personas que trabajan realizando gran trabajo muscular, y algunos postoperados y accidentados del tránsito. Las TAC tienen como objetivo en estos casos apoyar procesos de descontractura de partes blandas asociadas a sobre exigencia 
de la capacidad del tejido muscular agudo y crónico o a posiciones viciosas. La búsqueda del bienestar por otra parte se asocia a la verbalización del consultante de "no sentirse bien", "sentirse tenso" o "necesitar relajarse". En este caso, la reflexología y masoterapia pretenden contribuir al mejoramiento global del bienestar y a la reducción de la percepción de fatiga, y no a resolver dolores corporales específicos.

Importante resulta el diagnóstico de Enfermería en estas intervenciones. Ambas permiten realizar diagnósticos específicos, gracias al desarrollo de la sensibilidad de las manos, pudiendo detectarse contracturas musculares y puntos de tensión en los pies, que tendrían un significado de alteración de la normalidad para cada órgano en particular. Al mismo tiempo, la verbalización del cliente de malestar o dolor complementa el diagnóstico y prepara para la intervención tanto de relajación como terapéutica.

En términos de la intervención, se hará mención primero a su preparación y luego a las diferencias identificadas de acuerdo a grupos de personas consultantes. La preparación del ambiente para la realización de masoterapia y reflexología es un componente central del éxito de la intervención, pues apoya el proceso de relajación de la persona y la creación de un ambiente de confianza y privacidad. Se destaca la preparación y utilización de aceites naturales para aplicar durante el masaje, la musicoterapia de relajación (de preferencia sonidos de la naturaleza como viento o agua), la temperatura entre 22 y 24 grados Celsius, la aromaterapia con esencias florales y el uso de luz tenue y de colores suaves, como azul o violeta. La combinación de estos elementos ha permitido lograr que personas visiblemente tensas o ansiosas alcancen una buena relajación física y mental.

En cuanto a características particulares de quienes solicitan estas terapias, se han observado diferencias según etapa del ciclo vital. De esta manera, niños y adolescentes demoran más tiempo en lograr la relajación y la utilización de imaginería apoya el proceso; además, el tiempo total de intervención tiende a ser más breve y suele acompañarse de conversación, resolviendo inquietudes en torno a la preparación del ambiente, que reduce la ansiedad asociada al proceso. Los adultos mayores, en cambio, manifiestan inseguridad frente a la exposición del propio cuerpo, prefiriendo por este motivo reflexología o masajes de extremidades o cabeza. La mayoría de ellos busca mejorar la sensación de bienestar y son especialmente receptivos al trato centrado en sus necesidades individuales. Respecto del masaje, existe una tendencia a solicitar masaje suave o digitopuntura. De acuerdo a la experiencia con adultos, por otra parte, se aprecian diferencias entre mujeres y hombres. Mientras que las mujeres en general conocen más del tema y se realizan con mayor frecuencia masoterapia y reflexología, los varones asisten con mayor frecuencia secundaria a alguna lesión o por recomendación médica. Éstos últimos se muestran más reservados a comentar el uso de estas terapias, quizás por un componente cultural que asocia en el hombre la palabra "masaje" con un escenario erótico o de insinuación sexual, o de preocupación excesiva de la apariencia física, pero hace falta investigar en torno a esta temática. En cuanto al dolor como motivo de consulta, las mujeres refieren malestar en extremidades inferiores por várices, cefaleas y dolor de espalda a nivel lumbar. Los hombres, por el contrario, refieren generalmente dolor de espalda a nivel cervical y ciático. Como principal aspecto en común, tanto varones como mujeres asisten a estas terapias por trastornos del ánimo, siendo la relajación física y mental un objetivo en sí mismo y referido como de alto impacto. La oportunidad que ofrece en este sentido la reflexología y masoterapia es vivida como "un espacio para mí... para el descanso y la reflexión", de autocuidado, encuentro personal y sanación.

El profesional de Enfermería es exigido y 
desafiado en la práctica de estas intervenciones, pues demandan una atención centrada en cada individuo, respondiendo a sus necesidades, inquietudes y expectativas particulares. Además, la búsqueda del "sentirse mejor" dice relación con elementos no sólo físicos, sino también con aspectos emocionales y espirituales.

Finalmente, en términos de la evaluación, existen dos factores esenciales que se entrelazan y combinan, uno es la percepción del individuo de alivio y mejoramiento de su bienestar tras la intervención y otro es la percepción de éxito del profesional de salud que realiza dicha intervención. Respecto de este último factor, es interesante notar que la evaluación resulta de una dinámica mutua entre el profesional y el consultante, cambiante a cada momento, y que culmina en la verbalización del usuario de su bienestar en combinación con la percepción del profesional de haber contribuido en dicho proceso. Ambos entonces son sanadores y sanados, en tanto cuidan al otro por medio del cumplimiento de las expectativas manifestadas en un principio, y en tanto ayudan al otro a cuidar de sí mismo mediante la entrega de herramientas de autocuidado y autocuración.

\section{EL CUIDADO ALTERNATIVO Y COMPLEMENTARIO CENTRADO EN LA PERSONA}

Las raíces de la práctica del cuidado centrado en la persona quedan fielmente descritas en palabras de Hipócrates (8):

"Es más importante conocer cuál tipo de persona tiene la enfermedad que cuál tipo de enfermedad tiene la persona".

A través del proceso de enfermería desarrollado para reflexología y masoterapia, se relevan conceptos esenciales, como son el cuidado centrado en la persona, el cuidado llamado "alternativo y complementario", el concepto de bienestar y el proceso de auto- curación. Cada una de estas ideas son necesarias para articular y completar el escenario que se establece cuando un profesional de Enfermería asume el desafío de desarrollar competencias en torno a TAC. Este escenario, además, se repite día a día en la atención de cada persona, que trae consigo su universo particular, su modelo de creencias, sus expectativas, intereses, necesidades y capacidades de autocuidado.

En términos mundiales y como reflejo de las necesidades emergentes en salud, uno de los principales objetivos sanitarios para el año 2010 es mejorar en forma simultánea tanto los años de vida saludable como su calidad de vida. Para ello, las terapias alternativas y complementarias consideran estrategias que pueden ayudar a los individuos a alcanzar esta meta (16) y una de sus principales fortalezas está en centrarse en las necesidades e intereses particulares de cada consultante (13).

En cuanto al concepto de cuidado centrado en la persona, ésta considera como base de su actuar las necesidades particulares del individuo, dejándose de lado las intervenciones de tipo estándar y observando al usuario como una persona, reconociendo su conocimiento y experiencias previas y permitiendo que éste tome decisiones en torno a su salud, de acuerdo a la información entregada y bajo la filosofía del autocuidado (D. Orem) que postula que toda persona es capaz de cuidar de sí y de aprender a cuidar de sí (17). De esta manera, el profesional de salud que aplica el cuidado centrado en la persona reconoce al otro y provee condiciones para su participación en el cuidado de su salud (18). Por otra parte, la creencia de una persona respecto del proceso de salud y enfermedad es fundamental para su autocuidado y puede influenciar las respuestas tanto conductuales como psicológicas relacionadas a su condición de salud. La percepción de autoeficacia -cuán capaz se siente una persona de alcanzar una meta propuesta y efectivamente alcanzarla- 
es también un importante mediador de salud y autocuración; y puede desarrollarse y fortalecerse en todos los individuos por medio de la entrega de información pertinente, oportuna y clara, la educación para su autocuidado y el establecimiento de una relación terapéutica, colaborativa y horizontal entre quien solicita el cuidado y quien entrega herramientas para alcanzar la curación (19).

Continuando con la idea de cuidado centrado en la persona, en términos de las TAC la misión de los profesionales de la salud es proporcionar a los usuarios el acceso a una información amplia de opciones para su condición, sin excluir ninguna modalidad o sistema. Se reconoce además que cada persona es un ser único y complejo, dotado de múltiples niveles que no solamente incluyen lo físico, pues son igualmente importantes los factores mentales, emocionales y espirituales, y que condicionan nuestra salud en forma particular. Por lo tanto, un tratamiento que puede ser eficaz para una persona puede no funcionar en absoluto en otra con las mismas condiciones.

Respecto del concepto de autocuración (del inglés self-healing), la definición más pertinente para el escenario de las TAC dice relación con la resolución de procesos de desviación de la salud. Un ejemplo claro es el Reiki, donde mediante la imposición de manos y entrega de energía se logran restablecer procesos y/o sistemas del organismo debilitados, exagerados o alterados. Pero esto no resulta por arte de magia, sino se obtiene a través de interesantes y complejas relaciones internas entre lo físico, lo psicológico y lo espiritual; relaciones que recién se estarían descubriendo y validando a los ojos de la medicina alopática. De esta forma, la autocuración respondería a un fenómeno vital donde la persona es capaz de cuidar de sí y de curarse a través de la utilización de algunas estrategias específicas como la afirmación, la visualización, la relajación y la oración, entre otras; y sería el principio básico existente detrás de cualquier tipo de medicina utilizada (2).

Para Enfermería, el proceso de cuidado ha sido descrito por diversos teoristas. Jean Watson por su parte, plantea que el proceso de cuidado es dinámico y transpersonal y que se establece cuando existe una relación de cuidado auténtica entre la enfermera y el cliente (20). Para las autoras, la reflexología y masoterapia resultan exitosas en la sanación del individuo y en el incremento de su bienestar por el hecho de ser un encuentro único, personal y siempre cambiante entre dos individuos, gracias al desarrollo progresivo de la confianza mutua y el respeto por el otro.

Los profesionales de la salud y en particular de Enfermería deben reconocer, incorporar y perfeccionar habilidades transversales para el proceso de cuidado alternativo centrado en la persona y promotor de la autocuración y del bienestar. Algunas de estas habilidades son capacidad de crítica y autocrítica, empoderamiento de los usuarios mediante el fortalecimiento de sus competencias individuales, capacidad de diálogo horizontal sin juzgar, reflexión ética, autonomía profesional, asertividad y entrega de información clara y completa (21). Estas habilidades se encuentran relevadas en la filosofía de existencia y utilización de las terapias alternativas y complementarias.

\section{IMPLICANCIAS PARA LA PRÁCTICA}

Los profesionales de Enfermería tienen frente a sí una oportunidad única de cuidado integral y centrado en la persona, gracias a la creciente incorporación de TAC en el proceso de cuidado convencional. Para ello, deben comprender todos los aspectos de este tipo de terapias, de manera de poder promover de manera efectiva las TAC para el mejoramiento del bienestar y calidad de vida de las personas (16).

Las terapias alternativas y complementa- 
rias invitan a la practica del cuidado centrado en la persona, en donde el terapeuta logra observar la realidad desde la perspectiva del consultante y comprender la experiencia del proceso de cuidado existente en cada persona (22). Además, conceptos transversales como cuidado alternativo y complementario, el concepto de bienestar y de autocuración, son componentes del cuidado que invitan a la consideración del individuo como ser único que trae consigo su universo particular, su modelo de creencias, sus expectativas, intereses, necesidades y capacidades de autocuidado.

De acuerdo a la experiencia en masoterapia y reflexología y junto con lo descrito en la literatura, se vislumbra la necesidad de incorporar herramientas clínicas concretas y sólidas de este tipo de terapias en los estudios de pregrado es decir en el currículo profesional formal en salud así como también deberían ser incluidas en programas de educación continua que constituyen una excelente instancia de formación y profundización en temáticas relevantes para la innovación y búsqueda de soluciones específicas en el quehacer del profesional de la salud. La masoterapia y reflexología son excelentes herramientas de cuidado preventivo y terapéutico, que desafían al profesional a invertir en nuevos conocimientos y competencias interesantes para la sociedad. Cada día con mayor frecuencia, las personas requieren y solicitan sentirse cuidadas por manos expertas, solícitas y centradas en su individualidad particular.

Finalmente, resulta necesario apoyar y fortalecer investigaciones en torno a esta área del cuidado, pues desde la experiencia brinda grandes satisfacciones y permite vislumbrar en forma incipiente su contribución en el proceso de cuidado de la persona. La validación científica, entonces, permitiría alcanzar escenarios diversos y contribuir al mejoramiento de la salud, al bienestar y a la promoción del autocuidado de la comunidad.

\section{REFERENCIAS}

1. Burke A, Upchurch D, Dye C, Chyu L. Acupuncture use in the United States: Findings from the National Health Interview Survey. The journal of alternative and complementary medicine. 2006;12(7): 639-648.

2. Robb WJ. Self-healing: a concept analysis. Nursing forum. 2006; 41(2):60-77.

3. Kim S, Erlen J, Kim K \& Sok K. Nursing Students' and Faculty Members' Knowledge of, Experience with, and Attitudes Toward Complementary and Alternative. Therapies Journal of Nursing Education. 2006; 45(9), 375-378.

4. Organización Mundial de la Salud. Estrategia de la OMS sobre medicina tradicional 2002-2005.

5. Organización Mundial de la Salud. Medicina tradicional, informe de la secretaría. 56 Asamblea mundial de la salud. OMS. 2003.

6. Asociación Española de Médicos $\mathrm{Na}$ turistas. Qué es la Medicina Naturista. 2008. Hallado en: http://www.medicosnaturistas.es/ [Acceso en 2010].

7. Moreno M. Medicinas Alternativas: descripción general. Ministerio de Sanidad y Consumo. $1^{\text {a }}$ Ed. Madrid, España: AETSA. 2000.

8. Borgerson K. Evidence-based alternative medicine? Perspectives in Biology and Medicine. 2005;48(4):502-516.

9. Fisher P \& Adam W. Medicine in Europe. Complementary medicine in Europe. BMJ 1994;309:107-111.

10. Avogo W, Frimpong J A, Rivers P A, Kim S S. The effects of health status on the utilization of complementary and alternative medicine. Health Education Journal 2008;67:258-275.

11. Laurenson M, MacDonald J, McCready T, Stimpson A. Student nurses' knowledge and attitudes toward CAM therapies. Bri- 
tish journal of nursing. 2006;15(11):612-5.

12. Rojas-Cooley MT, Grant M. Complementary and altenative medicine: oncology nurses' experiences, educational interests, and resources. Oncology nursing forum. 2006;33(3):581-8.

13. Fewell F, Mackrodt K. Awareness and practice of complementary therapies in hospital and community settings within Essex in the United Kingdom. Complementary therapies in clinical practice. 2006;11(2):130-6.

14. González JR. Introducción al proceso de atención de Enfermería. Escuela Universitaria Virgen de los Desamparados, Valencia. Hallado en aniorte.eresmas. com/.../trabaj_introducc_proces_enfermer.pdf [Acceso en enero 2010].

15. Roper N, Logan W, Tierney A J. Proceso de Atención de Enfermería. Madrid: Ed. Interamericana. 1985:7-25.

16. Fowler S, Newton L. Complementary and alternative therapies: the nurse's role. The Journal of neuroscience nursing : journal of the American Association of Neuroscience Nurses. 2006;38(4):261-4.
17. Cavanagh, S. Modelo de Orem, aplicación práctica. $1^{\text {a }}$ Ed. Barcelona, España: Ediciones Científicas y Técnicas. 1993.

18. Eldh AC, Ekman I, Ehnfors M. Conditions for patient participation and nonparticipation in health care. Nursing ethics. 2006;13(5):503-14.

19. Galland, L. Patient-centered care: antecedents, triggers, and mediators. Alternative therapies in health and medicine. 2006;12(4):62-70.

20. Marriner A, Railey M. Modelos y Teorías en Enfermería. 5a Ed. Madrid, España: Mosby. 2003.

21. Schoot T, Proot I, Legius M, Meulen $\mathrm{R}$, De Witte L. Client- centered home care: balancing between competing responsibilities. Clinical nursing research. 2006;15(4), 231-54.

22. Jamieson M, Krupa T, O'Riordan A, O'Connor D, Paterson M, Ball C, Wilcox S. 2006. Developing empathy as a foundation of client-centred practice: evaluation of a university curriculum initiative. Canadian journal of occupational therapy. $73(2), 76-85$. 